\title{
La necesidad de la validación en los procesos de geosimulación
}

\author{
Montserrat Gómez Delgado
}

\section{Resumen}

En la presente conferencia se pretende realizar una revisión sobre la evolución que han experimentado los procesos de simulaciones espaciales aplicadas a la planificación del territorio a partir de las TIG, hasta derivar en la creación de lo que podríamos considerar una nueva subdisciplina geográfica: la geosimulación. La intención es abrir una discusión sobre la necesidad y escasa puesta en práctica de procedimientos adecuados de validación que avalen la fiabilidad y robustez de los resultados de unos modelos que no pueden ser contrastados con datos reales, pues se trata de simulaciones prospectivas, en la mayoría de los casos óptimas o deseables, que pretenden controlar y aminorar las consecuencias negativas de las acciones del hombre sobre el territorio, ensayando procesos de planificación futura más sostenibles. Estos procedimientos deberían contar, al menos, con algún proceso que nos permita averiguar la estabilidad y robustez de los resultados arrojados por el modelo (análisis de sensibilidad) y, por otro, controlar la incertidumbre derivada de los posibles errores existentes en los datos espaciales de partida (análisis de incertidumbre).

Palabras clave: Geosimulación / Tecnologías de la Información Geográfica / escenarios de simulación / validación.

\section{Abstract}

A review of the evolution of the spatial simulations and Geographical Information Technologies in the field of land use planning is presented. It is necessary to open a discussion about the development of appropriated validation methods to reinforce the confidence on the results of the geosimulation processes. The focus of the conference is on those prospective simulation models which results cannot be verified with real data. It is important on those cases, at least, assure the model's robustness by measuring the output stability during model running (sensitivity analysis) and the exploration of the error effects in model results derived from uncertainty of input data (uncertainty analysis). 
Keywords: Geosimulation / Geographical Information Technologies / simulation scenarios / validation.

Montserrat Gómez Delgado (montserrat.gomez@uah.es). Unidad docente de Geografía (Dpto. de Geología y Geografía), Universidad de Alcalá, España. 


\section{LOS INICIOS...}

Algo lejos quedan ya aquellos tiempos en los que se establecía las tres etapas clásicas de evolución de los Sistemas de Información Geográfica (SIG): la etapa pionera, a finales de los 60 y años 70 del pasado siglo, con la aparición de los primeros SIG comerciales y la preocupación principal por la resolución de los problemas técnicos derivados de la gestión y almacenamiento de la información espacial. La siguiente etapa en la década de los 80 , que podríamos denominar "de comercialización", una etapa de gran expansión y crecimiento, en la que aparecen ya las grandes empresas relacionadas con la comercialización de los SIG y en la que se da solución a análisis más complejos, posibilitando el desarrollo de aplicaciones más sofisticadas. En la tercera etapa que comienza a finales del siglo XX y llegaría hasta el momento actual, los cambios que se producen son más rápidos y diversos, afectando considerablemente a los datos estrictamente hablando: elaboración, almacenamiento, gestión, visualización, difusión, comercialización, etc. y, por otro lado, registrándose avances en la capacidad de resolución de problemas espaciales.

En el primer caso, y de manera resumida, se ha producido un gran avance en la integración y creación de datos estándar y diseño de metadatos; se mejora la conversión e intercambio de formatos; se desarrollan definitivamente los procedimientos para posibilitar la integración de SIG e Internet (creciendo enormemente la utilización de lenguajes espaciales e hipermedia); comienza la organización de las bases de datos orientadas a objetos (aunque no se popularizan ni desarrollan como se esperaba en un principio) y se dan soluciones tímidas y no definitivas a la inclusión y representación de la componente temporal del dato geográfico (que sigue siendo una asignatura pendiente). Por otro lado, aumentan las funcionalidades (y expansión) de los SIG comerciales (sobre todo de los más tradicionales) y comienza la era del software libre que, en este campo, tampoco tiene la repercusión y desarrollo que se esperaba. Es verdad que ha habido iniciativas prometedoras, pero parece que, por lo menos en el caso español, la crisis ha provocado una ralentización del desarrollo de las mismas.

Atendiendo a las tradicionales funciones básicas de un SIG de "entrada de datos" y "representación de resultados", habría que hacer mención al enorme desarrollo de lo que algunos autores (Bosque Sendra et al., 2013) ya consideran como otras técnicas diferenciadas dentro de las Tecnologías de la Información Geográfica y que están desarrollándose vertiginosamente en los últimos años: la 
Geografía Voluntaria o participativa, liderada por los desarrollos de Google Map o Google Earth (Goodchild, 2007) y que tienen su continuación en iniciativas como Wikimapia u OpenStreetMap (entre otras) y la geotecnología móvil (mobile geospatial technologies) y los servicios basados en la localización (location-based services).

En el segundo campo, resumido como resolución de problemas espaciales, el avance ha sido también bastante importante. Obviamente también se ha tenido que recurrir a otras disciplinas, técnicas, etc. para poder llevar a cabo este tipo de tareas de forma más completa y apropiada. Resumiendo, hasta finales de los años 90 del pasado siglo no se producen las sinergias suficientes para provocar un verdadero avance de los SIG en esta funcionalidad básica y tremendamente importante. Es a partir de los años 90 cuando se pone de manifiesto las deficiencias de los SIG en esta materia y las mejoras básicas que eran necesarias para conseguir configurarse como una herramienta definitiva en la gestión y planificación del territorio (Bosque Sendra, 2001, Bosque Sendra, 2005). Con el desarrollo de otras disciplinas y, especialmente con el espectacular avance de la computación y la programación, se abren nuevas y muy interesantes posibilidades.

En este sentido, quizás la aparición de los Sistemas de Ayuda a la Decisión Espacial (SADE) fue una de las primeras respuestas a esas deficiencias (Carver, 1999; Bosque Sendra et al., 2000; Kingston et al., 2000). La solución, como en otros campos de aplicación de los SIG, ha sido la combinación e integración con otras disciplinas y técnicas, además del empleo de una buena dosis de programación.

Tras los SADE, y con el auge de la planificación participativa, aparecen los Sistemas de ayuda a la planificación (Planning Support Systems), entendidos como un conjunto de herramientas geo-tecnológicas que han sido desarrolladas para apoyar procesos de planificación pública o privada, a cualquier escala y dentro de cualquier contexto específico de planificación (Brail y Klosterman, 2001; Geertman y Stillwell, 2003). La participación pública en este tipo de procesos de toma de decisiones espaciales supone la inclusión de herramientas de apoyo que pretenden facilitar la interacción de los diferentes agentes implicados, la transmisión de datos, de conocimientos, así como la el manejo de técnicas espaciales complejas (procedimiento de análisis multicriterio, localización de equipamientos, elaboración de mapas especializados, etc.) , sin la necesidad del conocimiento experto de las mismas por parte de todos los usuarios. Las primeras experiencias se desarrollan 
en el ámbito de la planificación urbana, pero pronto se extienden a otros campos como la gestión del sector público, provisión de servicios sociales, proyectos participativos promovidos por organizaciones no gubernamentales, etc. (Bosque Sendra y Gómez Delgado, 2010). En Barton y otros (2005) podemos encontrar una revisión de ejemplos de esta nueva forma de entender y desarrollar la tarea planificadora denominada PP-GIS (Public Participation GIS), o de manera más amplia y genérica PP-GIT (Public Participation Geographic Information Technologies).

Estos avances serían soluciones parciales que surgen concretamente en el campo y la preocupación por la planificación del territorio. De manera más genérica podemos decir que el salto más importante que se produce para intentar solventar estas deficiencias de los SIG se materializa a través de una iniciativa surgida desde un grupo de expertos en TIG y en técnicas estadísticas y cuantitativas, que comienza a combinar el conocimiento geográfico con otras disciplinas como la inteligencia artificial y la informática, aportando a este campo el uso de otras técnicas como los autómatas celulares, los modelos basados en agentes, el modelado borroso, las redes neuronales, la programación genética, etc. (Bosque Sendra, 2005). Esta iniciativa conduce a la creación finalmente de lo que se considera como una nueva subdisciplina geográfica: la Geocomputación ${ }^{1}$, entendida como una forma alternativa de estudiar los problemas territoriales y que se define como "la aplicación de las técnicas y métodos de la informática para mostrar las propiedades espaciales, explicar fenómenos geográficos y resolver problemas geográficos" (Couclelis, 1998).

Paralelamente en estos últimos años, y ante el avance y la gravedad de los problemas derivados del cambio global en el planeta, se hace cada vez más necesaria la utilización de la simulación para intentar de alguna forma obtener imágenes de futuro que nos ilustren sobre las posibles consecuencias de la acción del hombre sobre el territorio. Así, "la simulación por ordenador aparece como una forma diferente y original de hacer ciencia, que no pretende tanto formular leyes y teorías generales, difíciles de establecer para los hechos sociales, sino de crear modelos matemáticos, lo más sencillos posibles, que reproduzcan de la mejor manera algún aspecto del comportamiento y de la organización del territorio" (Bosque Sendra, 2005: 11). De esta forma aparece una nueva era de modelar simulaciones

\footnotetext{
${ }^{1}$ Es posible encontrar más información en www.geocomputation.org. Esta comunidad organiza conferencias de manera ininterrumpida desde 1996. La próxima tendrá lugar en Mayo de 2013 en la Universidad china de Wuhan.
} 
espaciales a la que se denomina Geosimulación ${ }^{2}$, entendida ésta como el diseño y construcción de modelos espaciales de alta resolución basados en objetos que se utilizan para explorar ideas e hipótesis sobre cómo operan los sistemas espaciales (Benenson y Torrens, 2004). Esta nueva forma de hacer ciencia combina conceptos, métodos y técnicas de la Geografía (especialmente las Tecnologías de la Información Geográfica), las matemáticas, la computación y en cierta forma bebiendo las fuentes de la Teoría General de Sistemas. Si bien es cierto que esta nueva forma de entender la modelación se puede aplicar a un número importante de problemas y fenómenos espaciales, ha sido la simulación en el ámbito urbano donde se han realizado más avances y trabajos (Blecic et al., 2009).

\section{UN FUTURO PROMETEDOR: LA GEOSIMULACIÓN.}

El desarrollo de modelos bajo esta perspectiva está siendo muy importante en los últimos años y se augura un futuro muy prolífico, por ejemplo, con las posibilidades abiertas por los modelos basados en agentes. La representación detallada del medio a través de las bases de datos SIG y la teledetección es crucial y existe, por otro lado, una implicación importante de desarrollo y programación de software de simulación y herramientas de apoyo. La programación orientada a objetos y, por extensión, la modelación orientada a objetos (OOM, Object-oriented modeling), que combina elementos de la inteligencia artificial y los sistemas basados en el conocimiento (KBS, Knowledege-based systems), despuntan como técnicas especialmente útiles para estudiar sistemas dinámicos complejos.

Esta nueva familia de modelos que ha surgido al amparo de las nuevas técnicas y sus beneficiosas fusiones, rechazan la idea de realizar predicciones únicas y absolutas, a favor de la idea de crear numerosas visiones alternativas e identificar que pasaría en el caso de asumir ciertas decisiones sobre el devenir del territorio. Como es lógico, es en el ámbito de la planificación en general, y de la urbana en particular, donde más éxito ha tenido esta práctica, debido a lo complejo e incierto del fenómeno estudiado.

Así, la propuesta de escenarios, entendidos como visiones alternativas de una futura situación (Barredo et al., 2005) se convierten imprescindibles en este

\footnotetext{
${ }^{2}$ Es posible encontrar más información en la página web www.geosimulation.org/geosim, puesta en marcha por uno de los pioneros en este tipo de modelación: Paul M. Torrens
} 
contexto. La filosofía de los escenarios es investigar la implicación de diferentes perspectivas 0 alternativas de un desarrollo global, regional o local y crear una herramienta de visualización (Shearer, 2005). La ventaja de los escenarios es, por tanto, la posibilidad de recrear diferentes visiones de futuro, probables, posibles y/o deseables (pero no predicciones o previsiones en sentido estricto) y poder compararlas para aprender y tener una imagen de las consecuencias que tendrán en el territorio unas decisiones u otras. Esto permitirá trabajar con planes e instrumentos de planificación lo más adaptables y adecuados para una realidad altamente cambiante, dirigida por factores que en muchos casos escapan de su rango de actuación (Aguilera Benavente et al., 2010; Aguilera Benavente et al., 2011).

La aplicación de escenarios futuros materializado a través de modelos de simulación de crecimiento urbano, cambios de uso de suelo, etc., constituyen un enfoque que se puede denominar spatial scenario planning (Aguilera Benavente et al., 2010) y que implica el uso conjunto de instrumentos con imparable y creciente presencia en la literatura científica en los últimos años. Este enfoque espacial y de aplicación conjunta de modelos de simulación y escenarios en la planificación territorial, metropolitana y urbana, se ha convertido en una importante línea de investigación en el contexto del análisis y evaluación de cuestiones ambientales $y$ territoriales, y en general en las cuestiones relativas a los procesos de toma de decisiones en planificación.

Estos escenarios normalmente pretenden dar respuesta a preguntas como ¿qué pasará?, ¿qué puede pasar? o ¿qué debería pasar?, encuadrándose así en los tres modos de pensamiento futuro que los expertos suelen considerar: el predictivo, el exploratorio y el normativo (Aguilera Benavente et al. 2010). Obviamente no las tres orientaciones son apropiadas para tratar todos los fenómenos. En el campo que nos concierne, de planificación territorial y urbana, serían la exploratoria y la normativa las más apropiadas (figura 1). Estos escenarios, al contrario que el predictivo, se caracterizan por pensar y diseñar diferentes posibilidades de evolución futura que no tienen por qué coincidir con la más probable, evolución que en un enfoque predictivo se extrae de la relación causal probada entre determinados factores y el hecho proyectado a futuro, por lo que el objetivo es extraer un resultado y probar su validez. Ese enfoque resulta muy discutible y cuestionado por los expertos en el tratamiento de los procesos territoriales, pues se trata de fenómenos demasiado complejos y sujetos a las incertidumbres propias de las tomas de decisiones humanas que impiden realizar una predicción propiamente dicha. Así, movernos entre posibilidades (controladas o no por los planificadores) y situaciones 
deseables u óptimas, resultaría más acorde con las características de estos procesos.

Figura 1. Estrategias adaptadas de escenarios exploratorios y normativos.

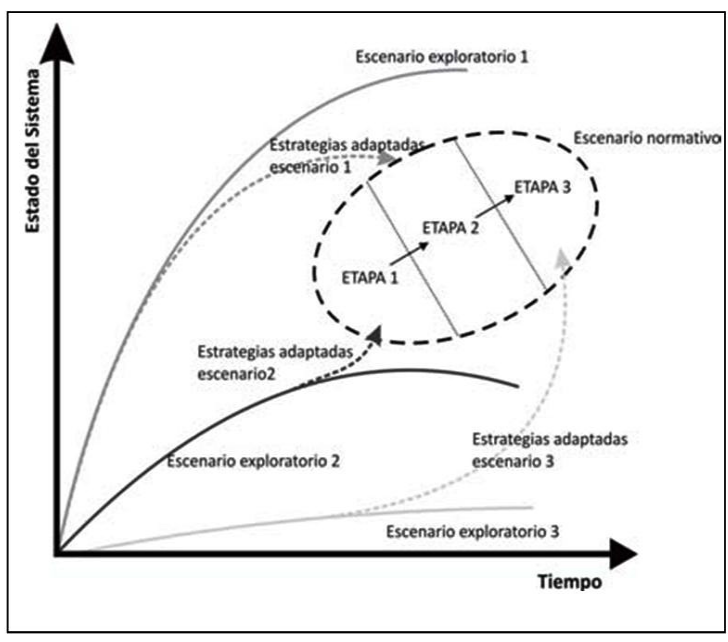

Fuente: Aguilera Benavente et al., 2011.

Una vez seleccionada la orientación de la simulación, el siguiente paso consistiría en diseñar el escenario de planificación que culminaría en una descripción más o menos detallada del mismo, conocida en la literatura anglosajona como storyline. En el trabajo que se viene citando de Aguilera Benavente y otros (2010) es posible encontrar información detallada sobre las fases y etapas a seguir para construir estos escenarios. Estos mismos autores identifican una serie de ventajas y mejoras que este proceso de spatial scenario planning aporta al proceso metodológico de planificación territorial, de los que serían destacables especialmente dos. En primer lugar, mejora la capacidad de adaptación de los planes, pues la propuesta de diferentes escenarios pueden adaptarse a la elaboración inicial del plan (adaptación ex ante) y, lo que es más importante, mejorar los efectos resultantes de la propia ejecución del plan (adaptación ex post). Por otro lado, mejora el proceso de comunicación y participación, ya que la propuesta de distintos escenarios puede ser la excusa perfecta para poner en contacto y discusión a los diferentes agentes implicados en el proceso de toma de decisiones territorial, que podrían aportar distintas perspectivas a la hora de elaborar el escenario más deseable. Este es uno de los objetivos principales de esa nueva forma de abordar la planificación denominado Public Participation GIS y al que nos hemos referido anteriormente. 
Estos escenarios, o base sobre los que se intentará crear esas hipotéticas imágenes de futuro, se han de materializar mediante algún proceso y técnica. Es decir, una vez establecidos los principios y desarrollado conceptualmente el escenario, es necesario simular la dinámica de ese fenómeno en el contexto establecido (figura 2).

Figura 2. Estructura de los escenarios.

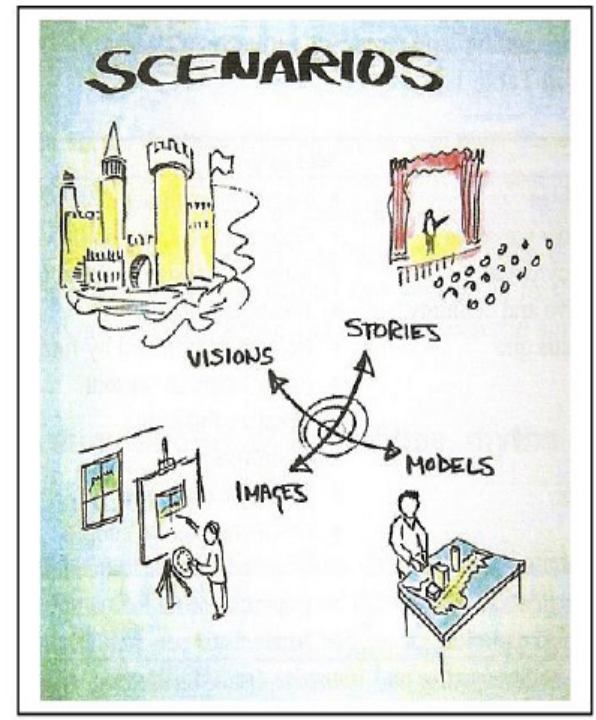

Fuente: Barredo et al., 2005.

Llegados a este punto sería apropiado hacer una aclaración. Si consultamos el diccionario de la lengua española, el significado de simular es "representar algo, fingiendo o imitando lo que no es". En este contexto cabe matizar que este ejercicio de simulación a partir de modelos no pretende imitar lo que no es, si no lo que podría ser. Así, la simulación es aquí considerada de forma general y genérica como la imitación de un proceso o sistema del mundo real a través del tiempo. Este acto de simulación requiere del desarrollo de un modelo que representará las características o comportamientos de ese proceso.

En nuestro contexto concreto, es necesario realizar una representación espacial de los escenarios diseñados, lo que les hace especiales por las variables a utilizar y la necesidad de utilizar técnicas que permitan reproducir las características del territorio. No se entiende ya que estas representaciones espaciales futuras en planificación se realicen de forma manual, a través de un diseño intuitivo. Actualmente los modelos de simulación, inicialmente desarrollados en el contexto 
de la revolución cuantitativa de la geografía, han experimentado un nuevo auge gracias al aumento y mejora de la computación, el desarrollo de las Tecnologías de la Información Geográfica y entre otros motivos, el extraordinario y descontrolado desarrollo de áreas urbanas en el planeta. Todo ello ha permitido el despegue definitivo de la Geosimulación.

Hoy en día encontramos numerosos ejemplos aplicados a los cambios de usos del suelo y en el ámbito de la planificación territorial. Los modelos y técnicas utilizadas son también muy diversos, como es posible comprobar en la obra de $H$. Briassoulis (2000). Si nos centramos en el ámbito de las aplicaciones explícitamente espaciales, y la combinación de las TIG con otras técnicas (es decir, el ámbito de la geosimulación), son las técnicas de Evaluación MultiCriterio (EMC), los Autómatas Celulares (AC) o cadenas de Markov los más exitosos, registrándose actualmente una incipiente utilización de los modelos basados en agentes para este fin (Carvalho, 2011).

Los modelos basados en AC han sido especialmente utilizados para la simulación espacial del crecimiento urbano, por su capacidad para representar las principales características de los procesos de dinámica urbana como la complejidad, autosimilitud, etc. Actualmente encontramos una cantidad ingente de trabajos en la literatura. En Santé et al., 2010 y en Triantakonstantis y Mountrakis (2012) es posible encontrar una amplia revisión de ellos. Actualmente encontramos más de 300 trabajos realizados en esta línea en los últimos 10 años, con lo que comprobamos el enorme éxito y proliferación de la geosimulación en éste ámbito de aplicación y otros relacionados con la planificación territorial.

Sin embargo, seguimos encontrando una gran deficiencia en la investigación, desarrollo y puesta en práctica de procedimientos que permitan validar los resultados de estas simulaciones.

\section{ASIGNATURA PENDIENTE: LA VALIDACIÓN}

Retrocediendo al uso estricto de los SIG en el ámbito de la planificación y la toma de decisiones, ya en la literatura se ha ido recogiendo desde los años 90 la dificultad que conlleva el control de la calidad de los datos en sí, la propagación de los errores a través de las distintas operaciones ejecutadas en un SIG o la validez de los resultados obtenidos a partir de la aplicación de las distintas funciones y operaciones disponibles en el mismo (Guptill y Morrison, 1995; Gómez Delgado y 
Bosque Sendra, 2004). Con la integración de otras técnicas y procedimientos, la necesidad de demostrar la calidad y validez de los resultados obtenidos se hace todavía más necesaria (Gómez Delgado y Bosque Sendra, 2009).

No debemos olvidar que un modelo es siempre una versión simplificada de la realidad que nos permite describir y comprender mejor un determinado problema, recogiendo los elementos y mecanismos esenciales de los sistemas del mundo real (tanto físicos como sociales, económicos o medioambientales). Sin embargo, y a pesar de las ventajas que en el ámbito de la planificación territorial estos modelos pueden presentar, un aspecto necesario en la elaboración de modelos de simulación es la demostración de que esos instrumentos generan representaciones fiables de los sistemas que imitan, teniendo siempre presente que sus resultados, en cualquier caso, no podrán coincidir exactamente con el sistema real que intentan reproducir.

Pero estos procesos de validación han de ser necesariamente distintos dependiendo del tipo de modelo tratado. En el caso de los modelos de simulación desarrollados en las ciencias experimentales, ingeniería, etc., el objetivo es certificar su capacidad operacional y, por tanto, es necesario aplicar procesos que verifiquen su grado de predicción. Sin embargo, en el ámbito de las ciencias sociales se desarrollan modelos de simulación con el objetivo de comprender un fenómeno determinado, corroborar ciertos aspectos teóricos (Rykiel, 1996) o, en el caso de la geosimulación, reproducir distintos tipos de escenarios futuros de desarrollo de un determinado fenómeno espacial. El problema crucial para Rykiel es decidir si el modelo es aceptable para el uso que se le pretende dar (por ejemplo, si imita los procesos del mundo real de manera aceptable) y qué confianza ofrecen las inferencias acerca de los sistemas reales que pueden derivarse de los resultados del modelo. Se trataría, por tanto, de averiguar la adecuación de datos, modelos y resultados al uso que pretendemos hacer de ellos, denominado en la literatura anglosajona como fitness for use (Guptill y Morrison, 1995).

En cualquier caso, no existe un acuerdo general en la comunidad científica sobre en qué debe consistir ese proceso de validación. Qureshi et al. (1999), por ejemplo, proponen un proceso formado por tres componentes:

a) Verificación de si el modelo está correctamente construido (desde el punto de vista formal). 
b) Validación de si la estructura del modelo representa adecuadamente el sistema modelado (si se ha construido el modelo correcto desde un punto de vista conceptual y operacional).

c) Aplicación de un análisis de sensibilidad que permita comprobar la robustez y estabilidad del modelo, examinando la variación que se produce en los resultados cuando se realizan variaciones sistemáticas en un rango de interés sobre uno o varios parámetros de entrada.

Otras propuestas dividen el proceso en tres tareas (Pagelow y Camacho Olmedo, 2008):

a) Verificación o validación interna para comprobar que el modelo está correctamente construido.

b) Calibración o comprobación de si el comportamiento global del modelo se ajusta a los objetivos. En el campo de la simulación, la calibración suele realizarse a partir de la comprobación del ajuste entre los resultados del fenómeno simulado y la situación real en un período previo.

c) Validación como medio para comprobar y mejorar la robustez y la aceptabilidad del modelo.

Sólo con estos dos ejemplos ya podemos comprobar cómo no hay un acuerdo en las componentes que deberían formar parte de este proceso de validación, ni en la definición o significado de las mismas. Es curioso, por otra parte, como se suele aludir a un proceso de validación en el que una de las partes también es denominada como validación. Esto nos parece confuso y poco atractivo para todo aquel científico que pretenda culminar su proceso de desarrollo e investigación con un ejercicio de validación. También hemos de destacar que en ningún caso se suelen abordar todas y cada una de las componentes mencionadas, pues el proceso se convierte en demasiado costoso en esfuerzo y tiempo, cuando ya se ha realizado una inversión importante de ambos en el desarrollo del modelo en sí. Quizás sería más recomendable y acertado intentar llevar a cabo al menos uno de ellos, de forma que los resultados del modelo resultaran relativamente fiables.

El significado literal de validar, según la Real Academia de la lengua Española, es dar fuerza o firmeza a algo, hacerlo válido. Por lo tanto, en este proceso se podría incluir cualquier procedimiento o procedimientos que nos permitan reforzar 
la credibilidad de los resultados obtenidos. Pero, ¿cuáles han de ser estos procedimientos?. Probablemente cualquiera que nos permita alcanzar este objetivo, dentro de la gama de posibilidades que actualmente podemos encontrar en la literatura, y en función del tipo de modelo a validar.

Hemos de mencionar que existen muchas propuestas para validar la geosimulación de cambios de usos del suelo. Las posibilidades son muy amplias cuando intentamos reproducir situaciones actuales con datos del pasado. Los resultados de esos cambios modelados pueden ser comparados con los datos actuales y, así, es posible medir su capacidad operacional de manera sencilla. En los últimos años encontramos una cantidad importante de aplicaciones y propuestas (Pontius y Schneider, 2001; Munroe et al.; 2002; Ménard y Marceau, 2005; Kocabas y Dragicevic, 2006; Burnicki, et al., 2007; Dendonker, et al., 2008, entre otros muchos). El problema es que gran parte de ellas (matrices de confusión, índice Kappa, etc.) no son viables cuando tratamos de simular la evolución de un fenómeno que no podemos contrastar con un dato real, aunque algunas propuestas sí que serían susceptibles de ser adaptadas a este tipo de modelos.

Sin embargo, al centrarnos en modelos de simulación de escenarios futuros para la planificación territorial, esto es, situaciones probables, óptimas o deseables, es necesario ampliar y flexibilizar este proceso de validación. Hemos de resaltar que, en estos casos, es imposible realizar una validación total, pues no existen datos reales con los que comparar los resultados obtenidos. Así, solo es posible abordar una validación parcial, que, como proponen Pagelow y Camacho Olmedo (2008: 29), podría realizarse contrastando los resultados con las opiniones de expertos, evaluando la estabilidad de los resultados o examinando si existe convergencia con los resultados de modelos diferentes.

Esta validación dependerá también del tipo de escenario simulado. Cuando se trata de representar a futuro tendencias que se han venido registrando hasta el momento actual, es muy habitual utilizar algunas de las técnicas mencionadas anteriormente para calibrar (ajustar) el modelo. Es decir, si con unas determinadas variables y configuración del modelo, la geosimulación es capaz de reproducir adecuadamente lo que sucedió desde el pasado hasta el momento actual, se considera que los resultados que este modelo arroje para el futuro serán igualmente válidos. Esta práctica ha sido muy desarrollada y perfeccionada especialmente en los modelos basados en AC. Aún así, sería necesario realizar algún tipo de análisis de los resultados a futuro que aportara algo más de información sobre la estabilidad 
y robustez de los mismos, sin embargo esta no es la práctica habitual.4

La cuestión se complica cuando tratamos de simular escenarios deseables, probables u óptimos. En este caso es necesario y posible realizar algún tipo de validación, adaptando algunos de los métodos ya existentes a las características particulares de este tipo de simulaciones. Es decir, no porque no dispongamos de datos reales con los que contrastar estos resultados, deberíamos conformarnos con lo arrojado por el modelo sin realizar un mínimo análisis de los resultados obtenidos.

Es cierto que en los últimos años se puede encontrar en la literatura más estudios sobre este tema, intentando hacer un esfuerzo por dar mayor confianza a los resultados de estos nuevos procesos de geosimulación, pero todavía queda mucho camino por recorrer, pues se trata siempre de propuestas parciales, sin aportar una metodología clara y cerrada del proceso de validación que debería llevarse a cabo.

En los últimos años, y tratando de poner ejemplos de diferentes propuestas y en diferentes tipos de modelos utilizados en la geosimulación (AC, cadenas de Markov, EMC), se ha estudiado el efecto de la propagación del error y la incertidumbre en los datos de partida (Yeh y Li, 2006; Burnicki et al., 2007; Fragkias y Seto, 2007); el efecto del tamaño del píxel y del proceso de rasterización (Jantz y Goetz, 2005; Dietzel y Clarke, 2006; Dendoncker et al., 2008); el efecto de la configuración, el tamaño de la vecindad y la escala en los modelos basados en AC (Ménard y Marceau, 2005; Samat, 2006; Kocabas y Dragicevic, 2009; Pan et al. 2010); el efecto del cálculo de la distancia en la técnica de EMC análisis al punto ideal (Baja et al., 2007); la incidencia de la variación de los pesos en un proceso de EMC (Chang et al., 2008, entre otros muchos). Sin embargo, escasos son los trabajos que, centrados en el proceso de geosimulación en sí, añaden una etapa final de validación de los resultados obtenidos (Baja et al., 2007; Fragkias y Seto, 2007; Hu y Lou 2007; Almeida et al. 2008;). Esta deficiencia es la que debería corregirse definitivamente y que los estudios no se centraran exclusivamente en este aspecto, sino que se considerase y tratase como una etapa más, y no menos importante, del desarrollo y puesta en práctica del modelo.

En este sentido, partiendo del significado literal del término validar, y teniendo en cuenta las múltiples posibilidades existentes para reforzar los resultados de un modelo, Gómez Delgado (2003) propone una metodología que pretende controlar el riesgo inherente a cualquier toma de decisiones basada en los resultados 
de un modelo explícitamente espacial y ejecutado en un entorno SIG (figura 3). Esta metodología se basa en el uso combinado de un análisis de sensibilidad (AS) y un análisis de incertidumbre (Al).

El AS, por un lado, nos permitiría estudiar cómo la variación de los resultados puede ser atribuida (cualitativa o cuantitativamente) a diferentes fuentes y cómo el modelo depende de la información de la que se nutre (Saltelli et al., 2000). Los resultados de estos análisis pueden indicarnos qué partes del modelo deberían ser mejoradas 0 a qué datos de partida deberíamos prestar especial atención por influir de manera más o menos importante en la variación de los resultados del mismo, de manera individual, o interactuando con otros. Inclusive los resultados de algunos de estos análisis pueden ser utilizados para realizar una simplificación del modelo, si queda demostrado que hay ciertos parámetros o variables que no tienen ninguna repercusión en la variación de los resultados (Gómez Delgado et al., 2012). Esta opción resulta muy interesante para optimizar recursos, especialmente en modelos como los aquí tratados, en los que hay que emplear un esfuerzo importante para recopilar toda la información espacial y temática necesaria para el buen funcionamiento de los mismos.

Figura 3. Validación en los procesos de geosimulación.

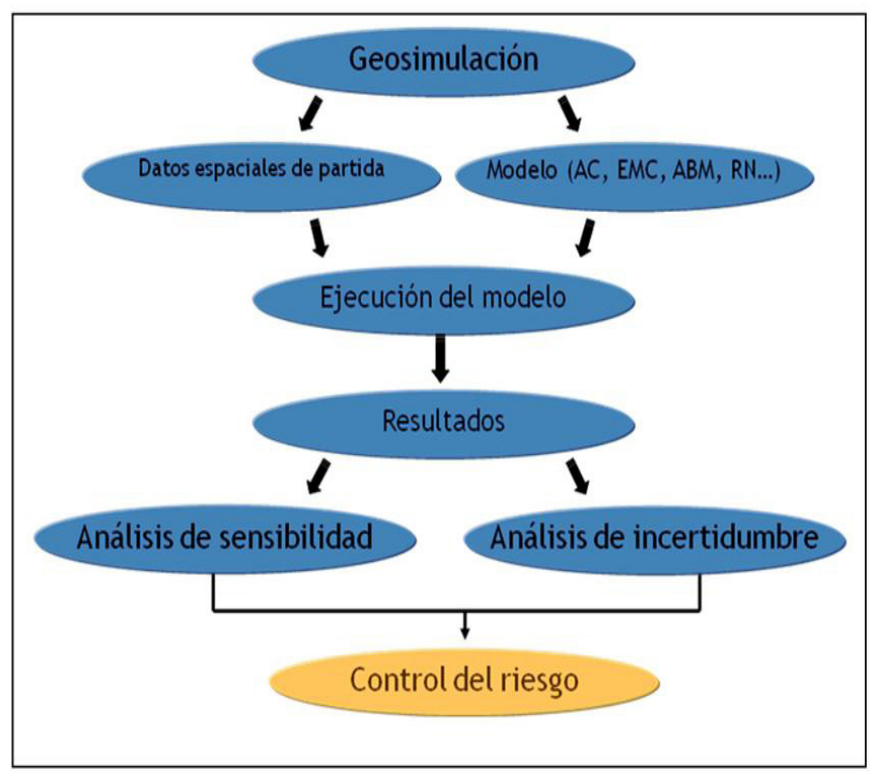


El Al, por su parte, trataría de ofrecer una alternativa a los procesos habituales de verificación que se relacionan exclusivamente con el problema del error en los datos de partida. Este proceso se ha demostrado insuficiente en el contexto de los modelos espaciales tratados en un SIG, por la falta de datos de mayor exactitud para realizar tales verificaciones, por las limitaciones para la estimación de la propagación del error (normalmente se asume que el error tiene una distribución normal y que no existe variación espacial del mismo) o la inexistencia de procedimientos y herramientas que permitan gestionar y reducir el error una vez estimado (Gómez Delgado y Bosque Sendra, 2004). En cualquier caso, se trata de un problema que actualmente es abordado en otros muchos campos, y así Fayerweather y otros (1999: 1077), desde las ciencias de la salud, advierten sobre cómo la comunidad científica se encuentra hoy dirigiendo sus esfuerzos hacia el desarrollo de procedimientos que nos permitan aproximarnos al riesgo de un modelo a través de la caracterización y cuantificación de la incertidumbre inherente a los resultados del mismo. Así, siguiendo el principio de validación que venimos proponiendo, el objetivo no sería tanto conocer la cantidad de error, sino poder tener la confianza de que el error y la incertidumbre a esos niveles no ponen en duda la validez de los resultados. Dado que uno de los principales problemas a la hora de examinar el problema del error es que no solemos contar con datos más exactos con los que llevar a cabo un proceso real de verificación, una de las posibilidades más habitualmente utilizadas es la de introducir algún tipo de error o perturbación en los datos de entrada del modelo e intentar cuantificar su impacto en la variación de los resultados del mismo (Gómez Delgado et al., 2012). A finales de los 90 encontrábamos en la literatura varias propuestas interesantes, aunque algunas de ellas difíciles de implementar (Emmi y Horton, 1996; Arbia y Haining, 1998). Basándose en estos trabajos previos, Gómez Delgado y Bosque Sendra (2004) realizan una propuesta más asequible de introducción de error utilizando las herramientas disponibles en cualquier SIG. En los últimos años, hemos podido comprobar cómo esta opción de validación ha sido muy popular, especialmente en la validación de modelos de cambios de usos del suelo (Yeh y Li, 2006; Burnicki et al., 2007; Rae et al., 2007; Dendoncker et al.; 2008; Pantus et al., 2008; Huang y Laffan, 2009).

De esta forma, pensamos que se abordan y tratan los dos problemas esenciales que pueden plantearse en estos ejercicios de geosimulación: por un lado el AS nos ofrecería información sobre la robustez de los resultados y el Al trataría uno de los principales problemas inherentes a cualquier dato geográfico: el problema del error y la incertidumbre. Por lo tanto, parece que como mínimo se deberían incorporar estos dos procesos a la etapa final de desarrollo de una geosimulación. 
Desde entonces se han desarrollado varios trabajos en esta línea, intentado realizar propuestas en ambos tipos de análisis (Gómez Delgado y Tarantola, 2006; Gómez Delgado y Bosque Sendra, 2009; Orán et al., 2010; Pascual et al., 2010; Orán et al., 2011; Pascual et al., 2011, Plata Rocha et al., 2012; Barreira González et al., 2012). En todos los casos se ha intentado adaptar la metodología 0 filosofía utilizada en procesos de validación ya consagrados y establecidos en otros campos disciplinares (modelado numérico utilizado en física, ingeniería, química, etc.) al contexto particular de la geosimulación, en el que las especiales características de las variables espaciales, supone todo un reto de adaptación, pero no imposible.

Esperamos que por lo menos esta breve conferencia sirva para concienciar más a los expertos y profesionales de la necesidad de incorporar estas prácticas en la geosimulación y animar a desarrollar metodologías y procedimientos adaptados a los distintos tipos de modelos que actualmente se utilizan para ofrecer nuevas perspectivas en el ámbito de la planificación. Sin duda será un valor añadido que reforzará la confianza de todos los agentes implicados en estos procesos, en los datos obtenidos a través de unas tecnologías con las que no suelen estar muy familiarizados.

\section{REFERENCIAS BIBLIOGRÁFICAS}

- Aguilera Benavente, F.; Gómez Delgado, M. y Cantergiani, C.C. (2010): "Instrumentos de simulación prospectiva de crecimiento urbano", en Ciudad y Territorio. Estudios Territoriales, Vol. 165-166, págs. 481-495.

- Aguilera Benavente, F.; Valenzuela Montes, L.M.; Soria Lara, J.A.; Gómez Delgado, M. y Plata Rocha, W. (2011): "Escenarios y modelos de simulación como instrumento en la planificación territorial y urbana", en Serie Geográfica, No. 17 , págs. 11-28.

- Almeida, C.M.; Gleriani, J.M.; Castejon, E.F. y Soares-Filho, B.S. (2008): “Using neural networks and cellular automata for modelling intra-urban land-use dynamics" en International Journal of Geographical Information Science, No. 22 (9), págs. 943-963.

- Arbia, G. y Haining, R. (1998): "Error propagation modelling in raster GIS: overlay operations", en International Journal of Geographical Information Science, 
Vol.12 (2), págs. 145-167.

- Baja, S.; Chapman, D.M. y Dragovich, D. (2007): "Spatial based compromise programming for multiple criteria decision making in land use planning" en Environmental Modeling \& Assessment, №. 12, págs. 171-184.

- Barredo, J.I.; Petrov, L.; Sagris, V.; Lavalle, C. y Genovese, E. (2005): "Towards an integrated scenario approach for spatial planning and natural hazards mitigation", Report EUR 21900 EN, Ispra, Institute for Environment and Sustainability (European Commission, Joint Research Centre).

- Barreira González, P.; Aguilera Benavente, F. y Gómez Delgado, M. (2012): "Propuesta de un análisis de sensibilidad para validar modelos prospectivos de simulación de crecimiento urbano basados en autómatas celulares", en GeoFocus, No.12, págs.. 303-328.

- Barton, J.; Plume, J. y Parolin, B. (2005): "Public participation in a spatial decision support system for public housing", en Computers, Environment and Urban Systems, №.29 (6), págs. 630-652.

- Benenson, I. y Torrens, P. (2004): Geosimulation: automata-based modelling or urban phenomena. London, John Wiley \& Sons.

- Blecic, I.; Cecchini, A. Trunfio, G.A. (2009): "A multi-agent Geosimulation infraestructure for planning", en Murgante, B.; Borruso, G. y Lapucci, A.: Geocomputation \& Urban Planning, Berlin, Springer, págs. 237-253.

- Bosque Sendra, J.; Gómez Delgado, M.; Moreno, A. y Dal Pozzo, F. (2000): "Hacia un sistema de ayuda a la decisión espacial para la localización de equipamientos", en Estudios Geográficos, Tomo LXI, No. 241, págs. 567-598.

- Bosque Sendra, J. (2001): "Planificación y gestión del territorio. De los SIG a los Sistemas de ayuda a la decisión espacial (SADE)" en El Campo de las Ciencias y las Artes, No. 138, págs. 137-174

- Bosque Sendra, J. (2005): "Espacio geográfico y ciencias sociales. Nuevas propuestas para el estudio del territorio", en Investigaciones regionales, No. 6 , págs. 203-224. 
- Bosque Sendra, J. y Gómez Delgado, M. (2010): "Sistemas de Ayuda a la Decisión Espacial para la ordenación del territorio”, en Pineda, M.C. y Bosque Sendra, J. (Eds): I Congreso Internacional de Ordenamiento Territorial y Tecnologías de la Información Geográfica, Servicio de Publicaciones de la Universidad de Alcalá, pp. 1-33.

- Bosque Sendra, J.; Aguilera Benavente, F.; Barreira González, P.; Gómez Delgado, M.; Rodríguez Espinosa, V.M. y Salado García, M.J. (2013): "La enseñanza de las Tecnologías de la Información Geográfica (TIG) en España y en Europa", XIV Conferencia Iberoamericana de Sistemas de Información Geográfica, Tegucigalpa (Honduras), 3-5 de Julio de 2013.

- Brail, R.K. y Klosterman, R.E. (2001): Planning support systems. Integrating geographic information systems, models and visualization, Redlands, CA, ESRI Press.

- Briassoulis, H. (2000). Analysis of land use change: theoretical and modeling approaches. Regional Research Institute, West Virginia University. [consulta 31-03-2013]. Disponible en http://www.rri.wvu.edu/WebBook/Briassoulis/contents.htm

- Burnicki, A.C.; Brown, D.G. y Goovaerts, P. (2007): "Simulating error propagation in land-cover change analysis: the implications of temporal dependence", en Computers, Environment and Urban Systems, Vol. 31(3), págs. 282-302.

- Carvalho Catnergiani, C. (2011): "Modelos basados en agentes aplicados a estudios urbanos: una aproximación teórica", en Serie Geográfica, No.17, págs. 107-121.

- Carver, S. (1999): "Developing Web-based GIS/MCE: improving access to data and spatial decision support tools", en Thill, J.C.: Spatial Multicriteria Decision Making and Analysis. A geographic information sciences approach, Aldershot, Ashgate Publishing Ltd., págs. 49-75.

- Couclelis, H. (1998):"Geocomputation and space", en Environment and Planning B: Planning and Design, No. 25th Anniversary Issue, págs. 41-47.

- Dendoncker, M.; Schmit, C. y Rounsevell, M. (2008): "Exploring spatial data 
uncertainties in land-use change scenarios", en International Journal of Geogra phical Information Science, Vol. 22(9), págs. 1013-1030.

- $\quad$ Dietzel, C. y Clarke, K.C. (2006): "The effect of disaggregating land use categories in cellular automata during model calibration and forecasting", Computers, Environment and Urban Systems, 30, pp. 78-101.

- Emmi, P.C. y Horton, C.A. (1996): "Seismic risk assessment, accuracy requirements, and GIS-based sensitivity analysis", en Goodchild, M.F; Steyaert, L.T. y Parks, B.O. (Eds.): GIS and Environmental modeling: progress and research issues. Fort Collins, CO: GIS World Books, págs. 191-195.

- Fayerweather, W.E.; Collins, J.J.; Schnatter, A.R.; Hearne, F.T.; Menning, R.A. y Reyner, D.P. (1999): "Quantifying uncertainty in a risk assessment using human data”, en Risk Analysis, Vol.19 (6), págs.1077-1090.

- Fragkias, M. y Seto, K.C. (2007): "Modeling urban growth in data-sparse environments: a new approach" en Environment and Planning B: Planning and Design, Vol.34 (5), págs. 858-883.

- Geertman, S. y Stillwell (Eds.) (2003): Planning support systems in practice. Berlin, Springer.

- Gómez Delgado, M. (2003): Sistemas de Información Geográfica y toma de decisiones: control del riesgo a partir de análisis de sensibilidad y análisis de incertidumbre. Tesis doctoral inédita, Universidad de Alcalá.

- Gómez Delgado, M. y Bosque Sendra, J. (2004): "Análisis de incertidumbre para la gestión del riesgo”, en GeoFocus (Artículos), No. 4, págs.179-208.

- Gómez Delgado, M. y Tarantola, S. (2006): "Global sensitivity analysis, GIS and multi-criteria evaluation for a sustainable planning of hazardous waste disposal site in Spain", en International Journal of Geographical Information Science, Vol.20, págs. 449-466.

- Gómez Delgado, M. y Bosque Sendra, J. (2009): "Validation of GIS-Performed analysis", en: Joshi, P. K., Pani, P., Mohapatra, S. N., Singh, T. P. (Ed.): Geoinformatics for Natural Resource Management, Nova Science Publishers, págs. 
$559-571$.

- Gómez Delgado, M.; Plata Rocha, W.; Orán Cáceres, J.P.; Calabia Aibar, A.; Barreira González, P. (2012): "Procedimientos de validación de modelos de crecimiento urbano futuro", en Gómez Delgado, M. y Rodríguez Espinosa, V.M. (Eds): Análisis de la dinámica urbana y simulación de escenarios de desarrollo futuro con Tecnologías de la Información Geográfica, Paracuellos de Jarama, Editorial Ra-Ma, págs. 237-274.

- Goodchild, M. (2007): "Citizens as sensors: the world of volunteered geography", en GeoJournal No. 69 (4), págs. 211-221. [consulta 31-03-2013]. Disponible en http://link.springer.com/article/10.1007\%2Fs10708-007-9111-y.

- Guptill, S.C. y Morrison, J.L. (1995): Elements of spatial data quality. Oxford, International Cartographic Association, Elsevier Science.

- Hu, Z. y Lo, C.P. (2007): "Modeling urban growth in Atlanta using logistic regression" en Computers, Environment and Urban Systems, Computers, Environment and Urban Systems, No. 31 (6), págs. 667-686.

- Huang, Z. y Laffan, S.W. (2009): "Sensitivity analysis of a decision tree classification to input data errors using a general Monte Carlo error sensitivity model", en International Journal of Geographical Information Science, Vol. 23 (11), págs. 1433-1452.

- Jantz, C.A. y Goetz, S.J. (2005): "Analysis of scale dependencies in an urban land use change model", International Journal of Geographical Information Science, 19 (2), pp. 217-241

- Kingston, R.; Carver, S.; Evans, A. y Turton, I. (2000): "Web-based public participation geographical information systems: an aid to local environmental decision-making", en Computers, Environment and Urban Systems, No. 24, págs. 109-125.

- Kocabas, V. y Dragicevic, S. (2006): "Assessing cellular automata model behaviour using a sensitivity analysis approach", en Computers, Environment and Urban Systems, Vol. 30, págs. 921-953. 
- Ménard, A. y Marceau, D.J. (2005): "Exploration of spatial scale sensitivity in geographic cellular automata", en Environment and Planning B: Planning and Design, Vol. 32, págs. 693-714.

- Munroe, D.K.; Southworth, J. y Tucker, C.M. (2002): "The dynamics of land-cover change in western Honduras: exploring spatial and temporal complexity", en Agricultural Economics, Vol. 27, págs. 355-369.

- Orán Cáceres, P.; Gómez Delgado, M. y Bosque Sendra, J. (2010). "Una propuesta complementaria de análisis de sensibilidad de un modelo basado en técnicas SIG y evaluación multicriterio", en Ojeda, J.; Pita, M.F. y Vallejo, I. (Eds). Tecnologías de la Información Geográfica: la Información Geográfica al servicio de los ciudadanos. Secretariado de Publicaciones de la Universidad de Sevilla. Sevilla, págs. 971-987.

- Orán Cáceres, P.; Gómez Delgado, M. y Plata Rocha, W. (2011): "Posibilidades de aplicación de un análisis de sensibilidad secuencial a un modelo basado en técnicas SIG y evaluación multicriterio", en Pineda Jaimes, N.B. et al. (Editores) (2011): La innovación geotecnológica como soporte para la toma de decisiones en el desarrollo territorial. XIII CONFIBSIG (Toluca, México, Mayo 2011), pp. $1-24$.

- Paegelow, M. y Camacho, M.T. (2008): Modelling Environmental Dynamics. Berlin, Springer-Verlag.

- Pan, Y.; Roth, A.; Yu, Z. y Doluschitz, R. (2010): "The impact of variation in scale on the behavior of cellular automata used for land use change modelling" en Computers, Environment and Urban Systems, Vol. 34, págs. 400-408.

- Pantus, F.J.; Ellis, N.; Possingham, H.P. y Venables, W. (2008): "Sensitivity analysis of spatially aggregated responses: A gradient-based method", International Journal of Geographical Information Science, Vol.22 (5), págs. 575-599.

- Pascual Rosa, V., Aguilera Benavente, F., Plata Rocha, W., Gómez Delgado, M. y Bosque Sendra, J. (2010): "Simulación de modelos de crecimiento urbano: métodos de comparación con los mapas reales". En: Ojeda, J., Pita, M.F. y Vallejo, I. (Eds.), Tecnologías de la Información Geográfica: La Información Geográfica al servicio de los ciudadanos. Secretariado de Publicaciones de la 
Universidad de Sevilla. Sevilla. págs. 1.000-1.013.

- Pascual Rosa, V.; Aguilera Benavente, F.; Barreira González, P.; Gómez Delgado, M.; Santos Preciado, J.M. y Bosque Sendra, J. (2011): "Métodos de comparación de mapas simulados del crecimiento urbano con mapas reales", en Pineda Jaimes, N.B. et al. (Editores) (2011): La innovación geotecnológica como soporte para la toma de decisiones en el desarrollo territorial. XIII CONFIBSIG (Toluca, México, Mayo 2011), págs. 1-19.

- Plata Rocha, W.; Gómez Delgado, M. y Bosque Sendra, J. (2012): "Proposal for the introduction of the spatial perspective in the application of global sensitivity analysis", en Journal of Geographic Information System, No. 4, págs. 503-513.

- Pontius, R.G. Jr. y Schneider, L.C. (2001): "Land-cover change model validation by an ROC method for the Ipswich watershed, Massachusetts, USA", en Agriculture, Ecosystems and Environment, Vol. 85, págs. 239-248.

- Qureshi, M.E.; Harrison, S.R. y Wegener, M.K. (1999): "Validation of multicriteria analysis models", en Agricultural Systems, Vol. 62, págs. 105-116.

- Rae, C.; Rothley, K. y Dragicevic, S. (2007): "Implications of error and uncertainty for an environmental planning scenario: a sensitivity analysis of GIS-based variables in a reserve design exercise", en Landscape and urban planning, Vol. 79, págs. 210-217.

- Rykiel, E.J. Jr. (1996): "Testing ecological models: the meaning of validation", en Ecological modelling, Vol. 90, págs. 229-244.

- Saltelli, A.; Chan, K. y Scott, E. (2000): Sensitivity Analysis. Chichester, John Wiley \& Sons, LTD.

- Samat, N. (2006): "Characterizing the scale sensitivity of the cellular automata simulated urban growth: a case study of Seberan Perai Region, Penang State, Malaysia", en Computers, Environment and Urban Systems, Vol.30, págs. 905920.

- Santé, I.; García, A.M.; Miranda, D. y Crescente, R., (2010): "Cellular autómata models for the simulation of real world urban processes: A review and analysis", 
en Landscape and Urban Planning, No. 96, págs. 108-122.

- Shearer, A.W. (2005): "Approaching scenario-based Studies: three perceptions about the future and considerations for landscape planning", en Environment and Planning B: Planning and Design, No.32, págs. 67-87.

- Triantakonstantis, D. y Mountrakis, G. (2012): "Urban growth prediction: a review of computational models and human perceptions", No. 4, págs. 555-587.

- Yeh, A.G. y Li, X. (2006): "Errors and uncertainties in urban cellular automata", Computers, Environment and Urban Systems, Vol.30, págs. 10-28. 